\title{
La sombra de Dante en Chile desde la Colonia hasta EL SIGLO XIX \\ José Blanco Jiménez
}

\section{Las razones de un título}

En algunas ocasiones, el filólogo debe partir de una hipótesis negativa.

Estaba convencido acerca de la escasa presencia de Dante en los primeros siglos de la historia de Chile, pero debía demostrarlo. De allí que tuve que revisar todas las fuentes disponibles acerca de la cultura y, en particular, de la literatura en mi país.El resultado fue, desgraciadamente, el que esperaba: el gran poeta trecentesco no era más que una sombra en el transcurso de los primeros 350 años de presencia europea en nuestro territorio.

\section{Las restricciones a los libros y a la imprenta}

El primer incunable registrado en España es de 1475 y las Cortes de Toledo, dos años después, legislaron para que se liberara de impuestos la circulación de libros en las posesiones españolas. Sin embargo, esta disposición duró poco porque fue eliminada con el establecimiento de la Inquisición y el predominio del clero, que defendía sus privilegios políticos. Se llegó así a prohibir la importación de libros sin permisos especiales. Los Reyes Católicos dispusieron que

ninguna obra, pequeña o grande, en latin o en castellano, se pudiese imprimir o vender, si era impresa afuera, sin licencia previa de las audiencias de Valladolid i Granada, los arzobispos respectivos en las ciudades de Toledo, Sevilla, Granada i Burgos i el obispo de Salamanca en la de Salamanca i Zamora. (Medina I XXIX)

Felipe II fue más allá y ordenó pena de muerte y confiscación de los bienes para los que imprimiesen o vendiesen obras impresas en otra parte sin la licencia real. Su sucesor, Felipe III, "mandó que ninguno de sus vasallos pudiese hacer publicar libros en otros reinos, so pena de perder 
la ciudadanía, empleos i dignidades, i la mitad de los bienes aplicada por tercias partes a la cámara, juez y denunciador" (Medina I XXX). Las medidas se incrementaron con Carlos II y los borbónicos "ilustrados" Felipe V, Fernando VI, Carlos III y Carlos IV: Este último permitió sólo la circulación del Diario de Madrid.

En América -como en todo el Imperio Español- la imprenta fue controlada política y culturalmente, por lo que el traspaso de la tradición oral a la palabra escrita fue muy pausado. De hecho, sólo a fines del siglo XIX la alfabetización permite la existencia de un público lector de libros y periódicos.

El primer testimonio de un libro impreso en América corresponde La Escala Espiritual para llegar al cielo, de San Juan Clímaco, publicado presuntamente en Méjico en 1537, pero del que no se conoce ejemplar alguno. La imprenta había llegado el año anterior. En Sudamérica, la imprenta debutó en Lima, donde Antonio Ricardo, un italiano venido de México, imprimió en 1584 la Doctrina christiana en quechua y aimará. Mucho más tarde los jesuitas publicarían varios libros de devoción y el Arte de la lengua guarani, del padre Antonio Ruiz de Montoya, en algunos pueblos de Paraguay, entre 1705 y 1727. Poco antes de su expulsión, en 1766, la Compañía habría de fundar una imprenta en su Colegio de Monserrat en Córdoba del Tucumán, que habría de ser trasladada a Buenos Aires una década más tarde.

Los libros habían hecho posible la difusión de las ideas de Independencia. Éstos llegaron en las maletas de los comerciantes o de los hijos de los criollos que iban a estudiar a Europa. Domingo Amunátegui Solar habla de tráfico de libros y señala que "el primer contrabandista fue el ilustre prócer don José Antonio de Rojas, quien, después de larga residencia en el Viejo Mundo, volvió a América en 1779" (1936; 17). Es así como, logrando burlar la censura real, introdujo en Chile la Enciclopedia y a los filósofos franceses.

Los chilenos no tuvieron una imprenta propiamente tal durante la Colonia. Sus casi inexistentes publicaciones fueron confeccionadas en Lima, de la que dependía económica e intelectualmente. Obras de un cierto respiro (como "relaciones" o crónicas), fueron impresas en España o permanecieron inéditas hasta el siglo XIX.

En la segunda mitad del siglo XVI abundaron las gramáticas y los vocabularios indígenas, necesarios para la evangelización. Luego 
vinieron las hagiografías y las crónicas. Con la llegada de los jesuitas del Sur de Alemania, en 1747, el padre Karl Haimbhausen habría traído herramientas para imprimir, pero no existe certeza de sus trabajos tipográficos. Tras la expulsión de la Compañía, en 1767, los materiales pasaron a la Universidad de San Felipe. A pesar de que se sabe que funcionó hasta 1802, no hay rastros de impresos de ese período. De 1776 es el Modo de ganar el Jubileo, al que siguieron los cinco reglamentos internos de la Recolección Dominicana, en 1783.

La Primera Junta Nacional de Gobierno, en 1810, habría intentado traer una imprenta desde Buenos Aires, pero -conjeturas aparte- el primer equipo tipográfico (técnicos incluidos) fue traído de Nueva York, al año siguiente, por el comerciante sueco Mateo Arnoldo Hoevel, que la vendió al gobierno liderado por José Miguel Carrera.

La importancia de su adquisición queda de manifiesto en las vibrantes palabras de Camilo Henríquez en el Prospecto de La Aurora de Chile, primer periódico nacional:

Está ya en nuestro poder el grande, el precioso instrumento de la ilustración universal, la imprenta. Los sanos principios, el conocimiento de nuestros eternos derechos, las verdades sólidas y útiles van a difundirse entre todas las clases del Estado. Todos sus pueblos van a consolarse con la frecuente noticia de las providencias paternales y de las miras liberales y patrióticas de un gobierno benéfico, próvido, infatigable y regenerador. La pureza y justicia de sus intenciones, la invariable firmeza de su generosa resolución, llegará sin desfigurarse por la calumnia hasta las extremidades de la tierra. Empezará a desaparecer nuestra nulidad política; se ira sintiendo nuestra existencia civil; se admirarán los esfuerzos de una administración sagaz y activa, y las maravillas de nuestra regeneración. La voz de la razón y de la verdad se oirán entre nosotros después del triste e insufrible silencio de tres siglos. ¡Ah! En aquellos siglos de opresión, de barbarie y tropelías, Sócrates, Platón, Tulio, Séneca, hubieran sido arrastrados a las prisiones, y los escritores más célebres de Inglaterra, de Francia, de Alemania hubieran perecido sin misericordia entre nosotros; ;Siglos de infamia y de llanto! La sabiduría os recordará con horror, y la humanidad llorará sobre vuestra memoria. (Aurora, prospecto) 
Los patriotas -a través de la Junta Gubernativa de 1813- decretaron la libertad de imprenta, mandaron abrir una escuela primaria por cada cincuenta habitantes y reemplazaron La Aurora de Chile por El Monitor Araucano. El guatemalteco Antonio José de Irisarri inició la publicación del primer periódico privado del país.

La historia tipográfica de América ha sido fichada y analizada por José Toribio Medina. De la revisión acuciosa que hice de todo ese material, resulta que ninguna obra de Dante fue publicada en el continente.

Pero, de todas maneras, ¿de cuáles ediciones habrían podido disponer los hispanoamericanos?

En su ya clásico estudio, Irving A. Leonard menciona apenas dos veces a Dante en un Registro de Luis de Padilla, redactado en 1600 en Sevilla. Se trata de un inventario de libros "que iban dirigidos en la nave La Trinidad a la provincia de Nueva España para entregar en el puerto de San Juan de Ulua a Martín de Ibarra" (303-333).

Limitándome a la Commedia, sabemos que la "editio princeps" fue terminada en Foligno por JOHANN NEUMEISTER de Mainz el 11 de abril de 1472 y que fue seguida por 14 incunables, 30 cinquecentinas, las 3 ediciones del siglo XVII, las 24 del siglo XVIII y las 399 del siglo XIX. Según mi fuente de información, desde siglo XVIII y las 399 del siglo XIX. Según mi fuente de información, desde 1901 a 1921 aparecieron otras 69, lo que hace un total de 541 hasta esa fecha (La raccolta). De estas ediciones, en Argentina se encuentran la veneciana de Ottaviano Scoto da Monza (1484) y la bresciana de Bonino dei Bonini (1487), ambas con el comentario de Cristoforo Landino. Con respecto a este último, apareció por primera vez en la florentina de Nicolò di Lorenzo della Magna (30 de Agosto de 1481) y fue, entre 1484 y 1497, reimpreso cinco veces en Venecia y una en Brescia. Hasta la aldina de Pietro Bembo (Venecia, Agosto de 1502) fue inseparable del texto de la Commedia. En todo caso, volvió a aparecer, siempre en Venecia, cinco veces entre 1507 y 1536.

Para los alcances de mi trabajo, el comentario de Landino tiene una particular importancia, porque -como ha anotado justamente Nicola Bottiglieri- la primera referencia a la Commedia en el Nuevo Mundo corresponde a cuanto dice Fernández de Oviedo en el Libro VII Capítulo VI de su Historia Natural de las Indias. Mencionando el hecho de que los indios se pintan la cara de rojo cuando van a la guerra, agrega: "Y no nos 
debemos maravillar de esto, porque cuando los Romanos vencían, iban sobre un carro de silla dorada sentados y con el vestido palmada arriba, y con el rostro teñido de rojo, imitando el elemento fuego, como escribe Cristóbal Landino exponiendo la Comedia de Dante" (Actas). El cronista puede haberlo consultado en España o en América, pero en todo caso fue en lengua italiana.

Hay que esperar la segunda mitad del siglo XVIII para que aparezca el primer corpus completo de las obras de Dante (Venezia, 1757-58). Sin grandes novedades, se repiten las ediciones hasta que aparece la primera verdadera edición crítica de la Commedia debida a Karl Witte, que consultó más de 400 ejemplares para su collatio.

Pero a la filología texual me referiré en otra sede, pues de ella nadie en Chile -salvo el infrascrito- se ha ocupado hasta el momento.

\section{Colegios y Universidades}

Hasta fines del siglo XVI no hubo colegios en Chile. Los primeros "maestros de enseñar niños" fueron españoles desterrados del Perú. Más adelante, toda la actividad intelectual estuvo a cargo de la Iglesia Católica Apostólica Romana. En 1561, el papa Pío IV creó la diócesis del Nuevo Extremo, como sufragánea de la de Lima, que incluía desde el desierto de Atacama al sur, más las regiones de Tucumán y Cuyo. En 1564, el mismo pontífice creó la diócesis de La Imperial y Tucumán fue segregada en 1570 por Pío V. La importancia de los obispos fue, sin embargo, muy limitada debido a que el clero regular estaba mejor organizado y a que asumían el cargo muy ancianos, si es que no morían antes de llegar al país.

Los mercedarios vinieron como capellanes (los primeros con Diego de Almagro, en 1536) y no fundaron convento de inmediato, se establecieron como Orden en 1542. Los dominicos llegaron en 1552, los franciscanos en 1553, los jesuitas en 1593 y los agustinos en 1595. La Compañía, además de una escuela de primeras letras, creó un curso de filosofía para estudiantes religiosos y seglares. En 1600 fundó el Colegio Máximo de San Miguel, donde se enseñaba gramática latina, filosofía y teología.

Paulo V, en 1619, autorizó el otorgamiento de títulos de bachilleres y licenciados a los conventos dominicos de las Indias que estuviesen a más de 200 millas de las universidades de México y Lima. Es así como, en 1622, comienza a funcionar la Universidad de Santo Tomás 
de Aquino, primera del Reino de Chile, con sus cátedras de Teología y Arte, preparando a chapetones y criollos para acceder a cargos civiles y eclesiásticos. Al caducar el privilegio en 1684, Inocencio XI determinó que podría seguir funcionando hasta que la ciudad de Santiago poseyera una universidad pública.

El 1623, Gregorio XV (el mismo que canonizó a Ignacio de Loyola, Francisco Javier, Felipe Neri y Teresa de Ávila) autorizó a los jesuitas, con la bula In eminenti, para fundar el Convictorio San Francisco Javier, que se puso como objetivo formar sacerdotes y misioneros para evangelizar a los indígenas. Contó para ello con clérigos de la Compañía venidos de Paraguay. Pero la sociedad requería de "disciplinas profanas", como las matemáticas, el derecho y la medicina. Considerando que abogados y médicos tenían que obtener sus títulos en la Universidad de San Marcos de Lima, el cabildo de Santiago solicitó al rey, en 1713, la creación de una universidad.

Con cédula dada en San Ildefonso, a 28 de julio de 1738, Felipe V concedió la Universidad Real de San Felipe. Su primer "claustro universitario" se celebró en 1747, pero las clases empezaron en 1757, cuando era gobernador Juan de Amat y Junient. Sus facultades fueron cuatro: Teología (filosofía aristotélica y escolástica), Derecho (justinianeo y canónico), Medicina (no tuvo alumnos por mucho tiempo y no incluía estudios de anatomía ni cirugía) y Matemáticas (no tuvo ni profesor ni alumnos). Todas las clases eran en latín. A pesar de los inconvenientes, tuvo la importancia de introducir maestros laicos y aquí vinieron a estudiar jóvenes argentinos (de Cuyo, Córdoba, Buenos Aires y Salta), más cercanos a las ideas europeas por su situación atlántica.

La creación dela Universidad Real conllevó la revocación dela facultad de entregar títulos y grados que tenían las universidades pontificias. Desapareció así la Universidad de Santo Tomás, pero el Convictorio Carolino siguió funcionando hasta la expulsión de los jesuitas, cuando sus bienes pasaron a formar parte de la Universidad de San Felipe.

Con la difusión del despotismo ilustrado, a fines del siglo XVIII, se crearon dos establecimientos: el Convictorio Carolino (1778), anexo y preparatorio para la Universidad, reemplazó al jesuítico Convictorio de San Francisco Javier; y la Academia de San Luis (1797) del filántropo Manuel de Salas, que se dedicó a preparar agrimensores, alarifes, maestros de minas y funcionarios del consulado. 
A comienzos de movimiento independentista, la ya citada Junta de Gobierno de 1813 fusionó los cuatro planteles en el Instituto Nacional, además de crear la Biblioteca Nacional, a la que ya me referiré. La Real Universidad de San Felipe siguió funcionando sólo para otorgar grados de bachiller a los futuros abogados, que habían cursado sus estudios en el Instituto: tampoco le pertenecía ya su biblioteca. El Seminario Conciliar sería reabierto bajo la advocación de los Ángeles Custodios, sólo en 1832, por el obispo Manuel Vicuña. Por su parte, los jesuitas volvieron al país en 1843 y en 1856 crearon el Colegio San Ignacio, que aún subsiste.

El Instituto Nacional fue cerrado durante la Reconquista y restablecido en 1819, enseñando teología (para los seminaristas) y legislación (para los futuros abogados). Las materias eran latín, filosofía, retórica, física y dibujo. La $3^{\text {a }}$ Clase correspondía a Lenguas y Literatura (Gramática castellana. Traducción de lengua francesa. Traducción de lengua inglesa. Principios de elocuencia, poesía y crítica), que podía servirse "por dos profesores, y aún por uno." (Aurora, no 19, p. 4). Es decir, un solo profesor habría podido encargarse del castellano, el francés y el inglés.

La justificación de la elección del estudio de estas lenguas figuraba ya en La Aurora de Chile del 25 de Junio de 1812:

Artículo $5^{\circ}$. Organización y Método de la clase $3^{\mathrm{a}}$ : Además de la corrección que da al propio lenguaje el conocimiento de su gramática, facilita la inteligencia de los otros idiomas. Por tanto, se explicará la gramática castellana.

Consta por experiencia, que los jóvenes se aplican con sumo gusto en muchos colegios a aprender la traducción francesa y los principios de bellas letras, sin que este estudio los distraiga de otros más serios y difíciles. Por tanto, y en consideración a la excelencia de las obras escritas en aquella lengua, se enseñará su traducción, y a hablarlo si es posible.

El Inglés es igualmente una lengua sabia, consagrada a la filosofía y a la profundidad del pensamiento. Se enseñará, pues, su traducción por principios.

Las bellas letras son la delicia de la imaginación; aumentan la sensibilidad; dan esplendor a los estados y acreditan una educación fina. Se enseñarán pues en el Instituto por principios; mas no se emprenderá su estudio, hasta que los alumnos sepan 
alguno de los idiomas sabios, que son de constitución, para evitar la distracción y superficialidad. (Aurora, nº 20, p. 1).

El texto habla por sí solo, pero no está demás remarcar dos puntos que me parecen importantes: primero, se debe estudiar lenguas extranjeras y bellas letras "sin que este estudio los distraiga de otros más serios y difíciles"; y segundo, la lengua más importante es el francés.

Este segundo punto resultaría explicable si se piensa que las ideas iluministas llegaron en francés a las colonias españolas americanas, pero hay que ir más allá: la clase dirigente consideraba como imprescindible mandar a sus hijos a París a vivir un tiempo (sino a estudiar, por lo menos a pasear). De allí su presencia -hasta el día de hoy- en la escuela media chilena. Como profesor de italiano, respiro por la herida al decir que los docentes de francés se convirtieron en una cofradía que impidió la difusión de otras lenguas. En la actualidad, el francés -lengua inútilsigue siendo una rémora del plan de estudios en mi país.

A mediados del siglo XIX, Chile era institucionalmente estable. La Constitución de 1833, promovida por Diego Portales, habría de regir por casi un siglo. El triunfo contra la Confederación Perú-Boliviana de Andrés de Santa Cruz y la explotación minera, las exportaciones de trigo y maíz a California y Australia (debido la fiebre del oro) aseguraron bienestar económico: el puerto de Valparaíso fue el principal del Pacífico hasta la inauguración del Canal de Panamá, en 1914.

Siguiendo las ideas iluministas, el Estado se preocupó de fomentar la enseñanza. De este período son las escuelas prácticas de agricultura, de minería, de preceptores y de artes y oficios. Todos estos esfuerzos convergieron en la creación de la Universidad de Chile, que fue inaugurada el 17 de septiembre de 1843. Su primer rector, el venezolano Andrés Bello, señaló en el solemne discurso de instalación:

Las ciencias y la literatura llevan en sí la recompensa de los trabajos y vigilias que se les consagran. No hablo de la gloria que ilustra las grandes conquistas científicas; no hablo de la aureola de inmortalidad que corona las obras del genio.... Ellas [las bellas letras] llevan el consuelo al lecho del enfermo, al asilo del proscrito, al calabozo, al cadalso. Sócrates, en vísperas de beber la cicuta, ilumina su cárcel con las más sublimes especulaciones que nos ha dejado la antigüedad gentílica 
sobre el porvenir de los destinos humanos. Dante compone en el destierro su Divina Comedia. [...] La instrucción literaria y científica es la fuente de donde la instrucción elemental se nutre y se vivifica; a la manera que en una sociedad bien organizada la riqueza de la clase más favorecida de la fortuna es el manantial de donde se deriva la subsistencia de las clases trabajadoras el bienestar del pueblo. (16-38)

Lo evidenciado en negrita es mío. Bello escribió un manual de literatura, pero de Dante no dijo nada más. En todo caso, le interesaba dejar en claro que la Universidad debería tal vez decir a los jóvenes poetas chilenos:

Si queréis que vuestro nombre no quede encarcelado entre la cordillera de los Andes y la mar del Sur, recinto demasiado estrecho para las aspiraciones generosas del talento; si queréis que os lea la posteridad, haced buenos estudios, principiando por el de la lengua nativa. Haced más; tratad asuntos dignos de vuestra patria y de la posteridad. Dejad los tonos muelles de la lira de Anacreonte y de Safo: la poesía del siglo XIX tiene una misión más alta. Que los grandes intereses de la humanidad os inspiren. Palpite en vuestras obras el sentimiento moral. Dígase cada uno de vosotros, al tomar la pluma: Sacerdote de las Musas, canto para las almas inocentes y puras. (Instalación).

El Plan de 1843 introdujo la historia y, en El Semanario de Santiago del 5 de enero de 1843, el sabio polaco Ignacio Domeyko pensaba que "con el tiempo el Gobierno no dejaría de agregar una quinta facultad de literatura i bellas artes, compuesta de las cátedras: De literatura antigua, de literatura moderna, de academia de pintura, i de academia de música." Pero las buenas intenciones estaban lejos de verse realizadas.

La Universidad de Chile -cuyo nombre era coherente con la nueva realidad republicana- se componía de cinco facultades y una de ellas era la de Filosofía y Humanidades, que debía también dirigir las escuelas primarias y ocuparse preferentemente de la lengua, la literatura, la historia y la estadística nacional. Es innegable que su rol fue básico en el desarrollo del país y abarcó prácticamente toda la labor intelectual del país, ya sea por iniciativa personal de sus miembros como actividad corporativa. En lo que se refiere a las letras, la actividad de la Facultad de Filosofía y Humanidades entre -1843 y 1879- permitió la revisión y 
aprobación de numerosos trabajos que fueron aprobados como textos de estudio o de apoyo a la educación. Ignacio Domeyko señala que sólo entre 1854 y 1875 se aprobó un 80\% de los 121 libros presentados: 17 para la enseñanza superior, 52 para la secundaria y el resto como textos y libros de lectura para escuelas primarias. El mayor desarrollo lo tuvieron las investigaciones históricas; la literatura universal siguió siendo el pariente pobre.

\section{Bibliotecas y librerías}

Medina recuerda como primera biblioteca la del oidor de Santiago, Benito María de la Mata Linares, en la segunda mitad del siglo XVIII, notable sobre todo por los documentos históricos. Por su parte, los jesuitas no habían logrado agrupar más de tres a cuatro mil volúmenes "i de ellos la inmensa mayoría era en latin i versaban sobre materias teolójicas" ( Medina I XXXVIII).

Walter Hanisch entrega antecedentes al respecto, señalando que en 1767- el Colegio Máximo tenía 6.200 volúmenes, el de Concepción 2.209, el de Chillán 467, el de San Fernando 338, el de La Serena 940, el de Copiapó 254 y el de Melipilla 225. A fines del siglo XVIII, los dominicos tenían unos 5.000, los franciscanos 2.970 en 1798, los agustinos 3.000 y los mercedarios menos de 1.000. Entre los particulares, el obispo de Santiago, Manuel Alday de Aspée poseía 2.508 y Valeriano de Ahumada otros 1.449 (107-108). Medina registra, en la biblioteca del convento de La Merced, 756 volúmenes: 16 tomos de Biblias; Santos Padres 23; Escolásticos 146; filosofía 21; moralistas 105; predicables 94; varios (sobre todo místicos) 128. De literatura había un Séneca, un Josefo, un De Officiis de Cicerón y las poesías castellanas de un tal Benavides (Medina I XL).

Un catálogo de libros importados en 1620, publicado en El Bibliófilo Chileno (1947-1952;37-39) no aporta nada, como tampoco una importación realizada en 1794 por Juan Enrique Rosales, que figura en la misma revista $(54,67,70)$. Se lee en La Aurora de Chile del jueves 19 de marzo de 1812 una exhortación para que los comerciantes internen libros al país:

Uno de los muchos modos con que el comercio promueve y favorece la literatura es la introducción de libros científicos, y generalmente útiles; harán pues un gran servicio a la patria los comerciantes, que hagan venir tantas obras preciosas, que nos faltan. Por ahora hay algunos jóvenes, que desean aprender 
el inglés, pero no se encuentran diccionarios, ni gramáticas inglesas, que se dice haber en Buenos Aires, y que se pueden encargar a Norte América. (Aurora, ad locum).

La Biblioteca Nacional, cuyo primer director fue Manuel de Salas, se creó con los casi 5.000 libros de la Real Universidad de San Felipe, pero no tuvo importancia hasta que el Gobierno adquirió la colección perteneciente a los herederos de Mariano Egaña. Sin embargo, los libros fueron puestos a disposición del público sólo en 1855 (Barros Arana XV 107n.)

La Aurora de Chile, el jueves 2 de Septiembre de 1813, se refería a las obras donadas a la Biblioteca Nacional y solicitaba otras para las cátedras del Instituto. No hay un solo libro en lengua italiana ni de autor italiano. He podido revisar, publicado en El Bibliófilo Chileno, el Catálogo de los libros de Manuel de Salas, adquiridos por el Estado chileno para la Biblioteca Nacional y redactado el 22 de agosto de 1832: hay sólo volúmenes de historia, jurídicos y clásicos latinos en lengua original y traducidos (16-20).

Hacia 1830 en el territorio nacional residía alrededor de un millón de habitantes. La primera librería se instaló en la Imprenta de El Mercurio de Valparaíso en 1833 y fue comprada, en 1838, por el español Santos Tornero, que después la bautizó Librería Española, instalando después en Santiago: existía aún en 1936 como Librería Central y era propiedad de Mariano Servat.

Lastarria, hablando del año 1836, señala que

la librería de entónces era escasísima i de precios exorbitantes. Formaban su fondo muchos libros ascéticos i de antigua literatura española, los mui usuales de derecho civil, que se pagaban a mas de su peso en plata, poquísimos de historia, ninguno de ciencias, i algunos tratados de ciencia jurídica i de política, como Montesquieu, Fritot, Bentham. Gottui Vatel; Filangieri, Becaria, Rousseau, Constant, Rivero i Salas. (Estudios, 41-42)

Gaetano Filangieri (1752-1788), noble napoletano, publicó ensayos en la Scienza della legislazione, que fue puesto en el Índice y lo más probable es que se conociera aquí en su traducción francesa. Lo mismo debe haber ocurrido con Cesare Beccaria (1738-1794), milanés amigo de los Enciclopedistas franceses, y su Dei delitti e delle pene. 
La Universidad requería de una biblioteca, ya que la del Instituto Nacional no era suficiente. El Consejo decidió adquirir libros para actualizar el acontecer literario y científico en Europa. La actual colocación de la biblioteca es posterior a 1889, cuando fue demolido el antiguo templo de San Diego de la orden de los franciscanos, comprado por el Gobierno. El inventario de ese año -que incluía la unión de la biblioteca de ese convento, la del Instituto y la universitaria -arrojó una cifra de 9.800 volúmenes, que se vio duplicada por la compra de los diez mil volúmenes de la Biblioteca Americana del argentino Gregorio Beeche. Agregando los libros de Joaquín Rodríguez y los de la sucesión Pedro Montt, se contaban 75.000 ejemplares en 1921.

\section{Literatura en Chile y Literatura Chilena}

La literatura da sus primeros pasos con un poema épico, que ensalza al pueblo mapuche para hacerlo digno rival de los, hasta entonces aparentemente, victoriosos españoles.

Alonso de Ercilla es un poeta-soldado que, volviendo a España, ordenó sus versos y publicó la primera parte de La Araucana en 1570, completando el poema ocho años más tarde. Es un documento histórico que declara su influencia italiana:

Amor de un juicio rústico y grosero

Rompe la dura y áspera corteza;

Produce ingenio y gusto verdadero

Y pone cualquier cosa en más fineza:

Dante, Ariosto, Petrarca y el Ibero

Amor los trujo a tanta delgadeza;

que la lengua más rica y más copiosa

Si no trata de amor es disgustosa. (XV, 2: 1-8)

Con respecto a ecos dantescos, se ha tratado mañosamente de encontrar algunos: "De allí llegó al famoso Biobio / el cual divide a Penco del Estado, / que del Nibequetén, copioso río, / y de otros viene al mar acompañado" (La Araucana I, 61:1-4)

El recuerdo de Inf. V, "su la marina dove'l Po discende / per aver pace co' seguaci sui" (98-99) resulta forzado, como asimismo este otro paso que suele citarse: "Los nuestros, del temor mas aguijados, / al entrar de la noche se hallaro / en la estrema ribera de Biobío / adonde pierde el nombre y ser de río" (La Araucana III, 3: 1-8) 
Dante hace decir estas palabras al moribundo y arrepentido Buonconte de Montefeltro cuando llega al torrente: «Oh!», rispuos'elli, «a piè del Casentino / traversa un'acqua c'ha nome l'Archiano, / che sovra l'Ermo nasce in Apennino. / Là 've 'l vocabol suo diventa vano, / arriva' io forato ne la gola, / fuggendo a piede e sanguinando il piano" . Pd. V 94-97). Asimismo, la pretendida paráfrasis de Pd. XVII: “Tu proverai sí come sa di sale / lo pane altrui, e come è duro calle / lo scendere e 'l salir l'altrui scale" (58-60) estaría en los versos: “¿Qué cosa puede haber más afrentosa, / que ser huéspedes toda nuestra vida?" (La Araucana, VII, 27: 5-6).

Los demás poetas del período (Pedro de Oña, Juan de Mendoza, Hernando Álvarez de Toledo, Núñez de Pineda y Bascunán, Fray Juan de Barrenechea y Albis, el padre López, el padre Escudero, Lorenzo Múgica) no conocen a Dante. Lo mismo ocurre con los cronistas Alonso de Góngora Marmolejo, Alonso de Ovalle y Pedro Mariño de Lobera (en una copia modificada por Bartolomé de Escobar).

José Victorino Lastarria habría de afirmar lapidariamente en 1842: “Durante la Colonia no rayó jamás la luz de la civilización en nuestro suelo" (Discurso 7-8).

\section{El movimiento literario de 1842}

El Discurso lastarriano fue, en realidad, una respuesta polémica a las críticas de extranjeros avecindados en Chile. Después del gaditano José Joaquín de Mora, vinieron los exiliados argentinos: Domingo Faustino Sarmiento escribía en El Mercurio de Valparaíso; Vicente Fidel López y Juan Bautista Alberdi lo hacían en la Revista de Valparaíso. Todos coincidían en considerar a los escritores chilenos poco originales. Es así como se constituyó la Sociedad Literaria, que se reunió 86 veces desde el 5 de marzo de 1842 hasta el $1^{\circ}$ de agosto de 1843 . Tuvo 41 socios y sus órganos de difusión fueron el Semanario de Santiago, El Crepúsculo, El Siglo y la Revista de Santiago.

La polémica literaria entre ambos grupos -que ya ha sido analizada por ilustres estudiosos, como Raúl Silva Castro (Panorama)- me interesa sólo en lo que tiene que ver con mi argumento. Habiendo leído todo lo disponible, puedo asegurar que las referencias a autores italianos es mínima. 
El citado López escribía:

Sigamos ahora el movimiento del romanticismo en Francia que es donde está para nosotros su cuna moderna. Dante, Shakespeare, Calderón, Klopstock y otros muchos poetas italianos, ingleses, españoles y alemanes corrieron de mano en mano como la moneda del tiempo. Con ellos se encontraba naturalmente a sus anchas el romanticismo moderno porque era la expresión palpitante de la época de que él tomaba sus inspiraciones. ("Clasicismo" 132-133)

Y agregaba:

A medida que se fue desenvolviendo el genio francés y el genio italiano, se fue desenvolviendo esa tendencia a la organización que siempre han dejado ver estos pueblos en los tiempos antiguos y en los tiempos modernos. Por lo que hace a Italia, ahí está el derecho romano y el derecho canónico; la república y el imperio en la antigüedad, las repúblicas y el pasado en la Edad Media, ahí está Virgilio, Dante y Tasso; y esto prueba la realidad de esta tendencia que existe en el genio italiano a la armonía, al orden, a la organización, a la unidad. ("Consideraciones" V)

Hay que considerar que el Reino de Italia no existía aún.

Y más adelante:

Nada tan natural como el que dominara en esta reacción de la literatura francesa, la imitación de la literatura de los pueblos que mejor habían representado a la Edad Media por sus costumbres y por sus ideas; que eran la Italia del tiempo de Dante, la Alemania y la Inglaterra, que tenía el inapreciable tesoro de Shakespeare. ("Consideraciones" VI)

Lastarria creía que no debían seguirse modelos extranjeros y que había que excavar en la realidad social:

Se dice que la literatura es la expresión de la sociedad, porque en efecto es el resorte que revela de una manera la más explícita las necesidades morales e intelectuales de los pueblos, es el cuadro en que están consignadas las ideas y pasiones, los 
gustos y, opiniones, la religión y las preocupaciones de toda una generación. Forman el teatro en que la literatura despliega sus brillantes galas, la cátedra desde donde anuncia el ministro sagrado las verdades civilizadoras de nuestra divina religión y las conminaciones y promesas del Omnipotente; la tribuna en que defiende el sacerdote del pueblo los fueros de la libertad y los dictados de la utilidad general; el asiento augusto del defensor de cuanto hay de estimable en la vida, el honor, la persona, las propiedades y la condición del ciudadano; la prensa periódica que ha llegado a hacerse el agente más activo del movimiento de la inteligencia, la salvaguardia de los derechos sociales, el azote poderoso que arrolla a los tiranos y los confunde en su ignorancia. La literatura, en fin, comprende entre sus cuantiosos materiales, las concepciones elevadas del filósofo y del jurista, las verdades irrecusables del matemático y del historiador, los desahogos de la correspondencia familiar, y los raptos, los éxtasis deliciosos del poeta." (Estudios 42)

Los representantes del Movimiento Literario de 1842 fueron historiadores y poetas, pero sus modelos -como casi todas las manifestaciones culturales de la segunda mitad del siglo XIX- fueron franceses: Hugo, Lamartine, de Musset. Hay también ecos de Byron, Schiller, Zorrilla y Espronceda. Es necesario recordar que la poesía italiano estaba ligada sobre todo al fenómeno risorgimentale, pero sus ecos no se hicieron sentir.

Más tarde, Lastarria mantendrá una amplia correspondencia con Sarmiento, pero no hablarán de poesía italiana.

\section{Hacia fines del siglo XIX}

Diego Barros Arana, además de su Historia General de Chile, compuso un volumen de Elementos de literatura. Historia literaria, "obra escrita para la enseñanza en el Instituto Nacional y aprobada por la Universidad" (Elementos portadilla). En ésta da algunas indicaciones acerca de los esfuerzos precedentes. Asegura que la historia literaria formó parte de los planes de estudio desde 1848 y que se empleó una traducción manuscrita de un artículo escrito por M. Philarète Chaslej para un suplemento de la Encyclopédie moderne de Courtin (publicada 
entre 1824 y 1832). Apareció con el título Esquise d'une histoire générale des inffluences littéraires, en un tomo publicado bajo la denominación de Études sur l'antiquité (1840), pero el mismo Barros Arana reconoce que "basta recorrer a la lijera ese corto artículo para convencerse de que no ha podido servir de testo elemental de historia literaria" (Elementos V 1).

Dedicó el Capítulo VII a la Literatura Italiana y, en el párrafo 5 (Elementos 190-194), entregó un buen resumen de la Divina Comedia, con un desafortunado lugar común: "Los defectos del poema del Dante nacen de su erudicion, de su propio jénio i de las ajitadas pasiones políticas de su siglo, cuyo retrato fiel ha trazado" (Elementos 193).

En su infatigable labor investigativa, Raúl Silva Castro (Romanticismo 139-150) identificó los siguientes nombres de escritores chilenos en el siglo XIX:

Mercedes Marín de Solar, 1804-66; Ventura Marín, 1806-77; Salvador Sanfuentes, 1817-60; Hermógenes de Irisarri, 1819-86; Manuel Blanco Cuartín, 1822-90; Floridor Rojas, 1822-91; Juan Bello, 1825-60; Manuel Antonio Matta, 1826-92; Eusebio Lillo, 1826-1910; José Antonio Torres, 1828-64; Luis Román, 1828-97; Guillermo Matta, 1829-99; Guillermo Blest Gana, 1829-1905; Valentín Magallanes, 1831-82; Rafael Santos, 1833-70; Policarpo Munizaga Varela, 1833-90; Rosario Orrego de Uribe, 1834-79; Adolfo Valderrama, 1834-1902; Martín José Lira, 183567; Domingo Arteaga Alemparte, 1835-80; Isidoro Errázuriz, 1835-98; Amelia Solar de Claro, 1836-1915; Benjamín Vicuña Solar, 1837-97; Luis Rodríguez Velasco, 1838-1919; Eduardo de la Barra, 1839-1900; Zorobabel Rodríguez, 1839-1901; Carlos Walker Martínez, 1842-1905; José Antonio Soffia, 1843-86; Enrique del Solar, 1844-93; Esteban Muñoz Donoso, 18441907; Manuel Antonio Hurtado, 1845-1902; Carlos Morla Vicuña, 18461901; Víctor Torres Arce, 1847-83; Rosendo Carrasco y Jelves, 1847-1906; Francisco A. Subercaseaux, 1847-1910; Rodolfo Vergara Antúnez, 18471914; Juan Rafael Allende, 1848-1909; Luis Montt, 1848-1909; Guillermo Puelma Tupper, 1851-95; Javier Vial Solar, 1852-1935; Pablo Garriga, 1853-93; Pedro Nolasco Préndez, 1853-1906; Adolfo Quirós, 1853-1910; Manuel Antonio Guerra, 1855-94; Francisco Concha Castillo, 1855-1927; Guillermo Jünemann, 1855-1938; Nicolasa Marambio de Montt, 18571924; Bernardo Solar Avaria, 1859-1929; Domingo Antonio Izquierdo, 1859-86; Fermín Solar Avaria, 1859-96; Roberto Lagos, 1860-1928; Luis Barros Méndez, 1861-1908; Leonardo Eliz, 1861-1939; Narciso Tondreau, 
1361-1949; Pedro Antonio González, 1863-1903; Juan José Julio y Elizalde, 1863-1934; Julio Vicuña Cifuentes, 1865-1936; Ricardo Fernández Montalva, 1866-99; Roberto Huneeus Gana, 1867-1929; Augusto Winter, 1868-1927; Gustavo Valledor Sánchez, 1868-1930; Egidio Poblete, 18681940; José Zapiola,1804-85;Vicente Pérez Rosales,1807-86;Carlos Bello, 1815-54; José Victorino Lastarria, 1817-88; Martín Palma, 1821-84; Wenceslao Vial Guzmán, 1822-64; Francisco Bilbao, 1823-65; Manuel Bilbao, 1829-95; Alberto Blest Gana, 1830-1920; Justo Arteaga Alemparte, 1834-82; Daniel Barros Grez, 1834-1904; José María Torres Arce, 1852-87; Moisés Vargas, 1843-98; Ramón Pacheco, 1845-88; Vicente Grez, 18471909; Rómulo Mandiola, 1848-81; Rafael Egaña, 1851-1923; Borja Orihuela Grez, 1854-91; Arturo Givovich, 1855-1905; Daniel Riquelme, 1857-1912; Pedro Nolasco Cruz, 1857-1939; Pedro Pablo Figueroa, 1857-1909; Senén Palacios, 1858-1927; Alberto del Solar, 1859-1921; Eduardo Poirier, 18601931; Emilio Vaisse, 1860-1935; Enrique Montt, 1861-1913; Ricardo Cruz Coke, 1861-1920; Anselmo Blanlot Holley, 1861-1920; Luis Adán Molina, 1861-1952; Carlos Luis Hübner, 1862-1911; Francisco Hederra Concha, 1863-1944; María Mercedes Vial de Ugarte, 1863-1942; José Martínez Cuadra, 1864-96; Alejandro Silva de la Fuente, 1865-1956; Jorge Huneeus Gana, 1866-1926; Luis Orrego Luco, 1866-1948; Baldomero Lillo, 18671923; Felipe Aparicio Sarabia, 1867-1945; María Luisa Fernández de García Huidobro, 1868-1938; Pedro Balmaceda Toro, 1868-89; Federico Gana, 1868-1926.

Mi exhaustiva investigación acerca de este verdadero catastro onomástico tampoco permitió encontrar temas o referencias dantescas. Existen también dos libros señeros acerca de la literatura chilena hasta comienzos del siglo XX: el de Jorge Huneeus Gana (Cuadro) y el de Amunátegui Solar (Bosquejo).

En el primer libro, que es muy completo, Dante no es mencionado jamás. En el segundo, se citan algunos versos de José Antonio Soffia y Argomedo (Santiago de Chile, 1844 - Bogotá, 1880), relativos a la muerte de su madre en el incendio de la Iglesia de la Compañía, acaecido el 8 de diciembre de 1863:

La noche horrible

Visión sin nombre que temblar hiciera

De Dante la tremenda fantasía;

En ascuas calcinado el templo ardía 
Cual si el averno en su interior se abriera. (Bosquejo 344)

Genérica referencia a "lo dantesco", que cierra esta desilusionante revisión histórico-literaria.

Mi revisión de bibliotecas, que ya publiqué con anterioridad (Ediciones), deja en claro que la obra de Dante era conocida y está presente en muchas colecciones privadas (sobre todo las ediciones ilustradas por Doré). Seguramente fue aludido en charlas o conversaciones de salón, pero su presencia en el mundo de la cultura no fue más que una sombra. 


\section{Bibliografía}

Aurora de Chile. Consultar en www.auroradechile.cl.

Amunátegui Solar, Domingo. El progreso intelectual y político de Chile. Santiago de Chile: Nascimento, 1936.

- Bosquejo histórico de la literatura chilena. Santiago de Chile: Imprenta Universitaria, 1920.

Barros Arana, Diego. Elementos de literatura. Historia literaria. Santiago de Chile: Librería Central de M. Servat, 1893.

. "Un decenio de la historia de Chile." Obras completas. Santiago de Chile: Pedro G. Ramírez, 1883, t. XV. 107n.

Bello, Andrés. Instalación de la Universidad. Santiago de Chile: Imprenta del Estado, 1843. El Discurso también fue publicado en los Anales de la Universidad de Chile, 1, 1843-1844, 140-152, y en las Obras completas, Tomo XXI, 3-21. Ahora es consultable en www.uchile.cl/portal/ presentacion/historia/4682/ discurso.inaugural.

Blanco J., José. "Ediciones y traducciones de "La Divina Comedia" en bibliotecas chilenas". Mapocho, 58 (2005): 333-347.

Hanisch, Walter. Historia de la Compañia de Jesús en Chile (1593-1955). Buenos Aires: Francisco de Aguirre, 1974. 107-108.

Huneeus Gana, Jorge. Cuadro histórico de la producción intelectual de Chile. Santiago de Chile: Biblioteca de Escritores de Chile, 1908.

La raccolta dantesca della biblioteca Evan Mackenzie: Con la cronologia delle edizioni della Divina Commedia. Prefazione di U.L.Morichini, Genova: tip. del Risparmio, 1923.

Lastarria, José Victorino. Estudios literarios. Primera serie. Santiago de Chile: Imprenta Barcelona, 1912.

. Discurso de incorporación de D.J. Victorio Lastarria a una Sociedad de Literatura de Santiago, en la sesión del tres de mayo de 1842. Valparaíso: Imprenta de M. Rivadeneyra, 1842. 
Leonard, Irving A. Los libros del Conquistador. México-Buenos Aires: F.C.E., 1953.

López, Vicente Fidel. "Clasicismo y Romanticismo". Revista de Valparaíso, 4 (mayo 1842), pp. 122-143.

. "Consideraciones sobre el romanticismo-V". Gaceta del Comercio (3 de agosto de 1842), publicación periódica.

"Consideraciones sobre el romanticismo - VI". Gaceta del Comercio (4 de agosto de 1842), publicación periódica.

Medina, José Toribio. Historia de la literatura colonial de Chile. Santiago de Chile: Imprenta del Mercurio, 1878.

Silva Castro, Raúl. "Romanticismo y literatura chilena". Atenea, 395 (enero-marzo 1962): 139-150.

- Panorama literario de Chile. Santiago de Chile: Editorial Universitaria, 1961. 